\title{
Deficiencia de vitamina $D$ en la práctica clínica pediátrica
}

\author{
Vitamin D deficiency in pediatric clinical practice
}

\author{
Dr. Gustavo Cediel ${ }^{a, b}$, Dra. Johanna Pacheco-Acosta ${ }^{c}$ y Dr. Carlos Castillo-Durán ${ }^{+c}$
}

\begin{abstract}
RESUMEN
La investigación sobre vitamina D sugiere roles que van más allá del metabolismo óseo.

Objetivo: Actualizar la información sobre la deficiencia de vitamina D (DVD) en trastornos clínicos pediátricos.

Métodos: Búsqueda en bibliotecas virtuales, en la que se priorizaron estudios clínicos, longitudinales y metaanálisis sobre DVD en edades pediátricas publicados en los últimos 20 años. Se utilizaron como descriptores de búsqueda "deficiencia de vitamina D", "niños y adolescentes" $y$, en inglés, "vitamin D deficiency", "children and adolescents".

Resultados: En la población pediátrica, la DVD se asocia a diversas patologías clínicas, como alteraciones óseas, insulinorresistencia, síndrome metabólico, infecciones del tracto respiratorio, asma y enfermedades autoinmunes. Además, se asocia a prematurez, obesidad, mala absorción, medicación anticonvulsivante y a particularidades de vida, como uso de vestimentas, latitudes extremas, bajo consumo y poca exposición solar.

Conclusiones: La evidencia sugiere una alta prevalencia de DVD en varios trastornos y enfermedades en edades pediátricas. Se recomienda prevenir la DVD en las condiciones de riesgo, manteniendo concentraciones séricas de $25(\mathrm{OH}) \mathrm{D}>75 \mathrm{nmol} / \mathrm{L}$.

Palabras clave: vitamina $D$, deficiencia, revisión, niño.
\end{abstract}

a. PhD en Nutrición y Alimentos, Universidad de Chile.

b. Instituto de Nutrición y Tecnología de los Alimentos (INTA), Universidad de Chile.

c. Departamento de Pediatría, Facultad de Medicina Campus Centro, Universidad de Chile.

\section{Correspondencia:}

Dr. Gustavo Cediel: gcediel@inta.uchile.cl

Financiamiento:

Ninguno.

Conflicto de intereses: Ninguno que declarar.

Recibido: 3-2-2017

Aceptado: 6-7-2017 http: / / dx.doi.org/10.5546/ aap.2018.e75

Texto completo en inglés:

http: / / dx.doi.org/10.5546/ aap.2018.eng.e75

Cómo citar: Cediel G, Pacheco-Acosta J, Castillo-Durán C. Deficiencia de vitamina D en la práctica clínica pediátrica. Arch Argent Pediatr 2018;116(1):e75-e81.

\section{INTRODUCCIÓN}

La investigación en vitamina $\mathrm{D}$ (VD) ha tenido un protagonismo creciente debido a lo siguiente: 1) la caracterización del receptor (nuclear y citosólico) y la maquinaria enzimática que metaboliza la vitamina en múltiples tejidos (ej.: adiposo, músculo y páncreas); ${ }^{1}$ 2) el rol relacionado con la regulación de más de 200 genes $^{2}$ y 3) el riesgo asociado del rango subóptimo de las concentraciones séricas de 25-hidroxivitamina D [25(OH)D] con la presencia de múltiples enfermedades. ${ }^{3}$ Como consecuencia, en el área de la pediatría, es de gran interés discutir los roles extraóseos de la VD y tener en cuenta las medidas necesarias para evitar situaciones de deficiencia de VD (DVD).

El objetivo de esta revisión fue realizar una actualización de la información respecto a la DVD en algunos trastornos clínicos en edades pediátricas. La búsqueda se realizó en las bibliotecas on line Pubmed, Scielo y en referencias de otras revisiones. Los descriptores de búsqueda fueron "deficiencia de vitamina D", "niños y adolescentes" y los respectivos en inglés "vitamin D deficiency", "children and adolescents". Los criterios de inclusión fueron artículos publicados en los últimos 20 años, en los que se priorizaron ensayos clínicos aleatorizados y controlados, casos y controles y metaanálisis.

\section{Deficiencia de vitamina $D$ en niños y adolescentes}

En la última década, ha reaparecido el raquitismo por $\mathrm{DVD}^{4}$ asociado, principalmente, al bajo consumo de alimentos fuente y a la baja exposición al sol (invierno, vestimenta y/o uso excesivo de bloqueador solar). 5,6 Los estudios disponibles en población pediátrica muestran datos de $25(\mathrm{OH})$ $\mathrm{D}$ sérica que oscilan en el rango entre $24,5 \mathrm{nmol} / \mathrm{L}$ (Ushuaia, Argentina) y $116 \mathrm{nmol} / \mathrm{L}$ (Teherán, Irán). ${ }^{7}$ La magnitud del problema en niños y adolescentes en América Latina es desconocida; solo México presenta datos representativos nacionales, que 
muestran entre un $54 \%$ y un $28 \%$ de prevalencia de déficit/insuficiencia de VD en preescolares y escolares, respectivamente. ${ }^{8}$ Argentina presenta datos representativos en la región de la Patagonia en niños entre los 6 y los 23 meses, que muestran una concentración media de $25(\mathrm{OH}) \mathrm{D}$ sérica de $67,5 \mathrm{nmol} / \mathrm{L}$ (IC 95\%: 65,3-69,8), con valores extremos entre 10,5 y 177,5 nmol/L. Según se observa, los valores correspondientes al grupo de provincias relevadas en los meses más próximos al invierno (Chubut, Neuquén y Santa Cruz) son significativamente más bajos que en aquellas relevadas entre los meses de noviembre y diciembre (Río Negro, Tierra del Fuego y La Pampa). ${ }^{9}$ Algunos países han reportado prevalencia de DVD en niños con muestras no representativas, tales como Colombia del 10-12\% $(<50 \mathrm{nmol} / \mathrm{L})$, Brasil del 9\% $(<50 \mathrm{nmol} / \mathrm{L})^{8} \mathrm{y}$, recientemente, Chile del $64 \%(<50 \mathrm{nmol} / \mathrm{L})$ en preescolares de zonas australes $\left(>45^{\circ} 35^{`} \mathrm{~S}\right) .^{10}$ Esta evidencia sugiere una alta prevalencia de deficiencia e insuficiencia de VD en la región en niños y adolescentes, especialmente, durante el invierno y en latitudes extremas.

\section{Clínica de la deficiencia de vitamina $\mathrm{D}$ en niños y adolescentes}

Múltiples estudios han asociado las bajas concentraciones séricas de $25(\mathrm{OH}) \mathrm{D}$ con la presencia de enfermedades extraóseas en niños. ${ }^{3}$ A continuación, se describe la evidencia disponible sobre la relación entre condiciones clínicas y la DVD en edades pediátricas (Tabla 1).

Prematurez y DVD: El tercer trimestre de gestación es el período más relevante en términos de almacenamiento de masa mineral ósea y, por lo tanto, un período en el que aumenta el riesgo de alteraciones óseas por el déficit de VD. ${ }^{11} \mathrm{Un}$ estudio reciente evaluó a 100 niños de entre 23 y 27 semanas de edad gestacional (EG), con una media de peso de $770 \mathrm{~g}$, a los que dieron diferentes dosis de VD. A los 28 días de vida, presentaban DVD un $41 \%$ del grupo placebo, $16 \%$ del grupo que había recibido 200 UI diarias y $0 \%$ del grupo con 800 UI diarias. ${ }^{12}$ Esta evidencia coincide con la recomendación de la European Society for Paediatric Gastroenterology Hepatology and Nutrition (ESPGHAN), con aportes de 800 a 1000 UI de VD diarios, calculados a partir de alimentación y suplemento oral. ${ }^{13}$

Obesidad y DVD: La evidencia en niños prepúberes muestra una relación inversa entre las concentraciones séricas de 25(OH)D e indicadores de adiposidad, ${ }^{14,15}$ que es consistente con la hipótesis del secuestro de la VD por el tejido adiposo, dada su naturaleza liposoluble. ${ }^{16}$ Además, estos resultados coinciden con un estudio reciente que mostraba que el exceso de peso en niños influía en el suplemento farmacológico de VD y lograba una menor alza de $25(\mathrm{OH}) \mathrm{D}$; por lo tanto, los niños con exceso de peso podrían requerir dosis mayores de VD

TABLA 1. Recomendación de suplemento de vitamina $D$ en diferentes patologías en edades pediátricas

\begin{tabular}{|c|c|}
\hline Patología & Recomendación \\
\hline Obesidad & Suplir VD3 entre 1200 y 2000 UI/ día. ${ }^{18}$ \\
\hline Resistencia a la insulina & $\begin{array}{l}\text { Administrar } 4000 \mathrm{UI} / \text { día de VD3 por } 6 \text { meses en niños y adolescentes obesos con } \\
\text { déficit de VD. }{ }^{20}\end{array}$ \\
\hline Síndrome metabólico & Faltan estudios que clarifiquen las dosis y tiempos adecuados de VD para encontrar efectos. \\
\hline Diabetes tipo 1 & Administrar 2000 UI/ día de VD3. ${ }^{38}$ \\
\hline Fibrosis quística & 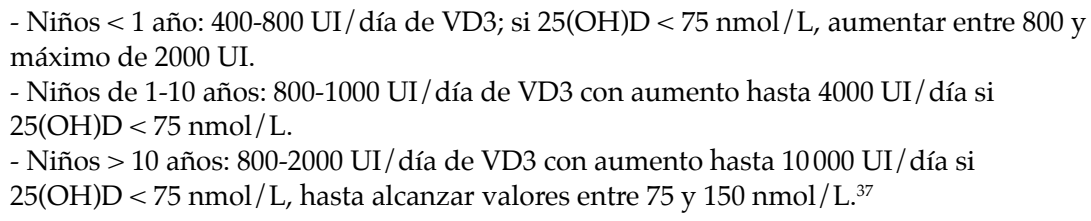 \\
\hline Enfermedad celíaca & $\begin{array}{l}\text { Dieta libre de gluten. Suplir con } 400-600 \text { UI de VD3 al día y cumplir con el } \\
\text { requerimiento de calcio. }{ }^{40}\end{array}$ \\
\hline Infecciones del tracto respiratorio & $\begin{array}{l}\text { Faltan estudios que clarifiquen las dosis y tiempos adecuados de VD que se relacionen } \\
\text { con efectos benéficos. }\end{array}$ \\
\hline Asma & Falta evidencia fuerte que avale el suplemento de VD3 entre 500 y 1200 UI al día. ${ }^{56}$ \\
\hline Enfermedades neurológicas & Suplemento de VD3 entre 800 y 1000 UI/ día. ${ }^{41}$ \\
\hline
\end{tabular}

VD: vitamina D. 
que los niños eutróficos para lograr las mismas concentraciones de 25(OH)D. ${ }^{17}$ Considerando el efecto que tiene la adiposidad sobre la biodisponibilidad de VD, los países del centro de Europa han emitido recomendaciones específicas para niños y adolescentes obesos: suplemento de 1200-2000 UI por día (30-50 g/ día) dependiendo de la gravedad de la obesidad durante otoño e invierno; sin embargo, es recomendable durante todo el año si la síntesis cutánea no es suficiente durante el verano. ${ }^{18}$

La evidencia sugiere que la forma activa de la VD $\left[1,25(\mathrm{OH})_{2} \mathrm{D}\right]$ regula la transcripción de genes involucrados en la adipogénesis, la inflamación y la resistencia a la insulina en el tejido adiposo en pacientes con obesidad. ${ }^{19}$ Además, en el músculo y en el páncreas, la $1,25(\mathrm{OH})_{2} \mathrm{D}$ podría mejorar la insulinosensibilidad a través de la regulación del flujo de calcio en estos tejidos, controlando la secreción de insulina en la células $\beta$ pancreáticas e incrementando la expresión del receptor de insulina en los tejidos periféricos. ${ }^{1}$ Resultados recientes en niños y niñas prepúberes muestran una relación inversa leve entre las concentraciones séricas de 25(OH)D e indicadores de resistencia a la insulina, aun después de ajustar por adiposidad. El punto de corte de $75 \mathrm{nmol} / \mathrm{L}$ de $25(\mathrm{OH}) \mathrm{D}$ es el que mejor predice estas condiciones. ${ }^{14}$ Un estudio clínico controlado en niños y adolescentes obesos de entre 9 y 19 años encontró una disminución significativa en el modelo homeostático para evaluar la resistencia a la insulina (homeostatic model assessment of insulin resistance; HOMA-IR, por sus siglas en inglés) en el grupo tratado comparado con el grupo placebo después de administrar 4000 UI/ día de VD3 por 6 meses (grupo tratado: $-1,36$ vs. grupo placebo: $+1,2) .{ }^{20}$ Además, los conocimientos sobre la VD activa como hormona con acciones asociadas a la acción insulínica en niños se inician ya hace casi 20 años, y se encuentra que el suplemento de VD disminuye el riesgo de diabetes tipo $1 .^{21-25} \mathrm{La}$ correlación con la diabetes tipo 1 puede atribuirse a acciones antiinflamatorias sistémicas de la VD como agente inmunomodulador mediante la acción sobre las células dendríticas, diferenciación de células $\mathrm{T}$ e interferencia en la generación y acción de citoquinas. ${ }^{26}$

Sindrome metabólico y DVD: Un análisis transversal de la Encuesta Nacional de Salud y Nutrición de los Estados Unidos entre 2001 y 2004 encontró que las bajas concentraciones de VD en adolescentes presentaban una fuerte asociación con el síndrome metabólico, independiente de la adiposidad. ${ }^{27}$ Otro estudio en niños escolares indígenas de Argentina (grupo con mayor riesgo de dislipidemia) mostró una disminución en las concentraciones de lipoproteínas de baja densidad (low density lipoproteins cholesterol; LDL-C, por sus siglas en inglés) después del suplemento con VD (beta $=-0,41, p<0,01) .{ }^{28-30}$ Aunque los mecanismos biológicos involucrados en estas asociaciones no han sido totalmente dilucidados, la evidencia sugiere un efecto cardiovascular y reno protector de la VD a través de la supresión del sistema renina angiotensina aldosterona, que inhibe la calcificación vascular y la formación de la capa ateroesclerótica, además de su acción antiinflamatoria e inmunomoduladora. ${ }^{31-33}$

Enfermedades con malabsorción intestinal y DVD: La prevalencia de DVD en pacientes con síndromes de malabsorción intestinal, que incluyen fibrosis quística (FQ), enfermedad celíaca (EC), síndrome de intestino corto y enfermedad inflamatoria intestinal, es mayor que en la población general. La disminución de VD en estos pacientes se debe a varios factores, tales como la disminución de absorción de vitaminas liposolubles y la mayor expresión extrarrenal de CYP27B1 mediada por la inflamación y el hiperparatiroidismo secundario a la hipocalcemia en algunos pacientes; esto lleva a una mayor conversión de $25(\mathrm{OH}) \mathrm{D}$ en $1,25(\mathrm{OH})_{2} \mathrm{D}$ $\mathrm{y}$, por tanto, a unas concentraciones de $25(\mathrm{OH}) \mathrm{D}$ más bajas. ${ }^{34}$

FQ y DVD: La prevalencia de un estado inadecuado de VD en pacientes con FQ puede llegar a ser tan alta como el $95 \% .{ }^{35} \mathrm{El} 90 \%$ de la población que padece $\mathrm{FQ}$ tiene insuficiencia pancreática, la cual lleva a malabsorción de grasas $\mathrm{y}$, con ello, de vitaminas liposolubles, como la VD. Sin embargo, la DVD también se observa hasta en el $50 \%$ de los pacientes con FQ que tienen una función pancreática adecuada. ${ }^{36}$ En los últimos años, se han evaluado otros desenlaces diferentes a la salud ósea en relación con la VD en niños con FQ; en este sentido, hay estudios recientes que asocian concentraciones de $25(\mathrm{OH}) \mathrm{D}$ con función pulmonar y colonización bacteriana por $S$. aureus y Pseudomonas sp. ${ }^{36}$ Actualmente, la Cystic Fibrosis Foundation (Fundación para la Fibrosis Quística, EE. UU.) recomienda la medición de $25(\mathrm{OH}) \mathrm{D}$ trimestralmente y un ajuste de dosis para alcanzar el grado de suficiencia de $25(\mathrm{OH}) \mathrm{D}(>75 \mathrm{nmol} / \mathrm{L}) .^{37}$

EC y DVD: Las concentraciones de VD y calcio están disminuidas en la mayoría de los pacientes con EC no tratada. Esto se debe a varias causas: la malabsorción por daño epitelial-intestinal, la restricción de lácteos por la intolerancia a la 
lactosa asociada y / o la reducción de la expresión de la proteína de unión a calcio, regulada por VD. En cuanto a polimorfismos del receptor de VD, los estudios no han encontrado diferencias entre personas con EC y sanas. Tanpowpong y Camargo expusieron la hipótesis de que la DVD llevaba a una desregulación de la respuesta inmune, especialmente una disrupción de la integridad de la mucosa intestinal durante la presentación antigénica (gluten) y antígenos microbianos (infecciones gastrointestinales virales), lo que promovía un ambiente microbiano desfavorable en individuos con predisposición genética a desarrollar EC. De esta forma, la DVD durante un período crítico de la vida como el primer año podría aumentar el riesgo de desarrollar EC posteriormente. ${ }^{39}$ El objetivo es mantener concentraciones de $25(\mathrm{OH}) \mathrm{D}>$ $75 \mathrm{nmol} / \mathrm{L} y$, para ello, el tratamiento principal es la dieta estricta libre de gluten, con lo cual se ha visto mejoría en las concentraciones séricas de VD y calcio, así como en la densidad mineral ósea en niños y adolescentes. ${ }^{40}$

Medicación anticonvulsivante y DVD: La prevalencia de DVD en niños con epilepsia y anticonvulsivantes es mayor del $50 \% .{ }^{41} \mathrm{La}$ fenitoína, el fenobarbital y la carbamazepina interfieren con el metabolismo de la VD. Estos fármacos actúan a nivel microsomal hepático induciendo la actividad de las enzimas hidroxilasas del citocromo P450, lo que lleva a una aceleración del catabolismo de la VD y sus metabolitos y a una disminución de su actividad; sin embargo, estos inductores enzimáticos son solo uno de los factores que se asocian a la DVD. Otros factores que se han asociado son la polifarmacia, duración del tratamiento anticonvulsivante $(79 \%$ de deficiencia e insuficiencia de VD con más de dos años de tratamiento), postración, alimentación por sonda nasogástrica o gastrostomía. ${ }^{41}$ Por lo anterior, se recomienda el suplemento con VD entre 800 y 1000 UI/ día en niños con enfermedad neurológica. ${ }^{41}$

Infecciones del tracto respiratorio y DVD: Durante el primer año de vida, hay un mayor riesgo de presentar bronquiolitis por virus respiratorio sincicial (VRS) cuando las concentraciones de VD en sangre de cordón son $<50 \mathrm{nmol} / \mathrm{L} .{ }^{42}$ Estudios realizados en Alemania por Łuczyńska et al., ${ }^{43}$ reportaron que el riesgo de infección respiratoria aguda baja (IRAB) aumentaba alrededor del doble en aquellos lactantes con concentraciones de $\mathrm{VD}<25 \mathrm{nmol} / \mathrm{L}$ en sangre de cordón. Además, parece haber una relación entre la DVD y la mayor gravedad de bronquiolitis por VRS. Esta disminución en la respuesta ante VRS parece estar asociada con el polimorfismo Fok 1 del receptor de VD y con una menor respuesta inflamatoria de las células epiteliales de la vía aérea mediada por VD. ${ }^{44}$ Asimismo, la VD ha sido referida como efectiva para disminuir el riesgo de influenza y para lograr una buena respuesta a la vacuna por activar células T. ${ }^{45,46}$ En nuestra búsqueda, no se encontró evidencia que soportara un efecto benéfico con el suplemento de VD para la prevención de infecciones respiratorias agudas, reducción de mortalidad o tasa de hospitalización por infecciones respiratorias. ${ }^{47}$

Asma y DVD: Varios estudios han asociado la DVD con la gravedad del asma. Einisman et al. ${ }^{48}$ no encontraron diferencias entre las concentraciones de $25(\mathrm{OH}) \mathrm{D}$ de niños saludables y asmáticos; sin embargo, dentro del grupo de niños asmáticos, encontraron diferencia en las concentraciones de suficiencia de $25(\mathrm{OH})$ $\mathrm{D}$, que fue mayor en los niños que estaban en fase 4 de tratamiento de asma según la Global Initiative for Asthma (GINA) y, en este mismo grupo, encontraron que el alelo $\mathrm{C}$ del Fok I del receptor de VD estaba presente en todos los niños a diferencia de los otros niños con asma. Un metaanálisis reciente evaluó estudios del suplemento de VD (las dosis usadas fueron de 500 y 1200 UI de VD3 al día) y mostró una reducción significativa en el riesgo de exacerbación de asma $\left(R R=0,28\right.$; IC 95\%: 0,12-0,64). ${ }^{49}$ Aun no se ha llegado a un consenso sobre las dosis y el tiempo para usar VD en niños con asma; sin embargo, la mayoría de la evidencia está más a favor del uso de dosis de VD entre 500 y 1200 UI de VD3 diarias, concomitante con el tratamiento estándar de asma.

Otros trastornos clínicos asociados a DVD: Estudios recientes muestran una asociación entre DVD y otros trastornos clínicos. Entre ellos, cabe destacar la asociación observada entre DVD y menarquía, la que estaba adelantada en 9 meses en niñas con DVD vs. aquellas con concentraciones normales, en Bogotá, Colombia. ${ }^{50}$ Se ha reportado en adolescentes una asociación inversa entre las concentraciones de $25(\mathrm{OH}) \mathrm{D}$ y presión sistólica. ${ }^{51}$ Además, hay estudios en los últimos años que muestran una posible asociación en algunas formas de cáncer, tanto en adultos como en niños. ${ }^{2}$ Futuros estudios podrán demostrar si el manejo de la deficiencia previene alguna de estas condiciones. 


\section{Prevención de la deficiencia de vitamina $D$ en niños y adolescentes}

La evidencia sugiere que la exposición a los rayos ultravioleta en una pequeña parte de la zona dorsal del cuerpo incrementa rápidamente las concentraciones de $25(\mathrm{OH}) \mathrm{D}$ en plasma hasta alcanzarse un plateau a los 15 minutos. ${ }^{52}$ Esto ha llevado a la recomendación poblacional de la exposición a la luz solar por 15 minutos, al menos, tres veces a la semana para cubrir los requerimientos de la vitamina; sin embargo, se requieren estudios similares en niños y adolescentes para validar esta sugerencia. En caso de ausencia a la exposición solar (ej.: pocas actividades al aire libre, condiciones climáticas, vestimenta o estacionalidad), la ingesta se convierte en la principal fuente de la vitamina. Debido a que son pocos los alimentos que contienen VD de manera suficiente para cubrir los requerimientos (Tabla 2) y que los contenidos de la vitamina varían en los alimentos dependiendo del método de preparación culinaria (ej.: en la fritura del pescado, se disminuye la VD en un 50\%), ${ }^{53}$ estrategias como el consumo de alimentos fortificados y la administración de 400 UI/ día durante el primer año de vida han probado ser costo-efectivas. ${ }^{15}$ Décadas atrás, a nivel mundial, se usaron dosis orales de 600000 UI para prevenir la DVD. Sin embargo, estudios posteriores encontraron una mayor probabilidad de efectos sobre el metabolismo del calcio, compromiso de talla y aumento de presión arterial. ${ }^{54}$ Estudios posteriores han mostrado que dosis entre 100000 y 150000 UI son capaces de prevenir la DVD, sin efectos adversos demostrados. ${ }^{55}$ En la Tabla 3, se muestran algunas recomendaciones para prevenir la DVD en población pediátrica.

\section{Tratamiento de la deficiencia de vitamina $D$}

La evidencia sugiere el suplemento de VD en los trastornos clínicos de riesgo de DVD mencionadas en esta revisión, para mantener concentraciones séricas de $25(\mathrm{OH}) \mathrm{D}>75 \mathrm{nmol} / \mathrm{L}$, punto de corte actual de suficiencia, con posibilidad de cambio a futuro (Tabla 1). En su mayoría, dosis entre 400 y 1000 UI de VD3 podrían ser suficientes para obtener estos valores; sin embargo, de no ser así, se deben hacer ajustes a las dosis, en lo posible, con control posterior de $25(\mathrm{OH}) \mathrm{D}$ hasta llegar a las

TABla 2. Contenido de vitamina $D$ en alimentos seleccionados

\begin{tabular}{lccccc}
\hline Alimento $^{*}$ & $\begin{array}{c}\text { Contenido } \\
\text { (UI/100 g o ml) }\end{array}$ & $\begin{array}{c}\text { Porción medida } \\
\text { casera (g o ml) }\end{array}$ & $\begin{array}{c}\text { Contenido } \\
\text { (UI/porción) }\end{array}$ & $\begin{array}{c}\text { \% de adecuación en } \\
\text { edad } \leq \mathbf{1} \text { año (400 UI) }\end{array}$ & $\begin{array}{c}\text { \% de adecuación en } \\
\text { edad > 1 año (600 UI) }\end{array}$ \\
\hline Salmón & 522 & Corte $(124 \mathrm{~g})$ & 647 & 161,8 & 107,8 \\
Jurel & 292 & Corte $3 \mathrm{oz}(85 \mathrm{~g})$ & 248 & 62 & 41,3 \\
Atún & 82 & Corte $3 \mathrm{oz}(85 \mathrm{~g})$ & 70 & 17,5 & 11,7 \\
Hígado & 49 & Rebanada $(68 \mathrm{~g})$ & 33 & 8,3 & 5,5 \\
Queso (tipo cheddar) & 24 & Rebanada $1 \mathrm{oz}(28 \mathrm{~g})$ & 7 & 2 & 1,2 \\
Huevo (yema) & 530 & Unidad $(17 \mathrm{~g})$ & 37 & 9,3 & 6,2 \\
Champiñones & 18 & Unidad $(19 \mathrm{~g})$ & 3 & 30 & 0,5 \\
Leche (fortificada) & 49 & Taza $(250 \mathrm{ml})$ & 120 & 30 \\
\hline
\end{tabular}

* Datos obtenidos del United States Department of Agriculture (USDA) por $100 \mathrm{~g}$ o ml y por porción.

$\mathrm{UI}=$ unidades internacionales $=0,025$ ug. Porcentaje de adecuación de vitamina D en niños $\leq 12$ meses, adultos $>71$ años y otros grupos según el Instituto de Medicina de los Estados Unidos: el requerimiento de vitamina D para lactantes de término hasta los 12 meses es de $400 \mathrm{UI}$; en personas mayores de 71 años, de $800 \mathrm{UI}$ y, en otros grupos, de 600 UI. ${ }^{57}$

\section{Tabla 3. Prevención de la deficiencia de vitamina $D$ en niños y adolescentes}

Para prevenir el déficit de vitamina D en la población pediátrica, se dan las siguientes recomendaciones:

- Exposición adecuada a la luz solar en la cara, las manos o las piernas (al menos, 3 veces a la semana por 15 minutos).

- En latitudes extremas y durante el invierno, se recomienda asegurar el consumo de alimentos fuente de la vitamina (Tabla 1).

- En lactantes, dar 400 UI de VD3 al día hasta el primer año de edad -por el bajo contenido de VD en la leche humana, $22 \mathrm{UI} / \mathrm{L}$ (de 15 a $50 \mathrm{UI} / \mathrm{L}$ ) - ${ }^{53}$ y, en latitudes consideradas extremas, analizar una posible mayor dosificación y extenderla hacia otras edades pediátricas. Analizar la posibilidad de reemplazo de dosis diarias por dosis únicas elevadas (100000 UI de VD) de dos a tres veces al año, en los casos en que haya dificultad para administrar dosis diaria por todo un año. ${ }^{58}$

- Prevención de sobrepeso y obesidad.

- Evaluar el estado nutricional de VD en las condiciones clínicas de riesgo de deficiencia y suplir de acuerdo con las recomendaciones (Tabla 1). 
concentraciones óptimas. Se sugiere que los niños con concentraciones de $25(\mathrm{OH}) \mathrm{D}<25 \mathrm{nmol} / \mathrm{L}$ sean remitidos a equipos especializados en el tema, para su estudio y manejo.

\section{CONCLUSIONES}

Los estudios disponibles sugieren que la deficiencia e insuficiencia de VD en la población pediátrica es alta. Hay varias funciones corporales que pueden verse afectadas por su deficiencia: metabolismos óseo, de la glucosa, de la inmunidad aguda, autoinmunidad, entre otras; los receptores nucleares de VD parecen también estar participando. Algunos trastornos clínicos que se asocian a DVD son obesidad, prematurez, período de lactancia, enfermedades con malabsorción intestinal, medicación anticonvulsivante; además, condiciones de vida, como uso de vestimentas que cubren todo el cuerpo, vivir en latitudes extremas, bajo consumo de alimentos fuente y poca exposición al sol.

Cada vez son más los trastornos clínicos que se están asociando a DVD, por lo cual se requieren más estudios para clarificar el rol de la VD en parámetros extraóseos en niños y adolescentes. Se recomienda prevenir y detectar tempranamente la DVD en las condiciones de riesgo y mantener concentraciones séricas de $25(\mathrm{OH}) \mathrm{D}>75 \mathrm{nmol} / \mathrm{L}$.

\section{Homenaje}

Manuscrito dedicado a la memoria del Doctor Carlos Castillo-Durán ${ }^{\dagger}$.

\section{REFERENCIAS}

1. MaestroB,MoleroS, BajoS, etal.Transcriptionalactivation of the human insulin receptor gene by 1,25-dihydroxyvitamin D(3). Cell Biochem Funct 2002;20(3):227-32.

2. Carlberg C, Molnár F. Vitamin D receptor signaling and its therapeutic implications: Genome-wide and structural view. Can J Physiol Pharmacol 2015;93(5):311-8.

3. Autier P, Boniol M, Pizot C, et al. Vitamin D status and ill health: a systematic review. Lancet Diabetes Endocrinol 2014;2(1):76-89.

4. Elder CJ, Bishop NJ. Rickets. Lancet 2014;383(9929):1665-76.

5 Society for Adolescent Health and Medicine. Recommended vitamin D intake and management of low vitamin D status in adolescents: a position statement of the society for adolescent health and medicine. J Adolesc Health 2013;52(6):801-3.

6 Lindqvist PG, Epstein E, Nielsen K, et al. Avoidance of sun exposure as a risk factor for major causes of death: a competing risk analysis of the Melanoma in Southern Sweden cohort. J Intern Med 2016;280(4):375-87.

7 Hilger J, Friedel A, Herr R, et al. A systematic review of vitamin D status in populations worldwide. $\mathrm{Br} J \mathrm{Nutr}$ 2014;111(1):23-45.

8 Brito A, Cori $\mathrm{H}$, Olivares $\mathrm{M}$, et al. Less than adequate vitamin D status and intake in Latin America and the Caribbean:a problem of unknown magnitude. Food Nutr Bull 2013;34(1):52-64.

9 Durán P, Mangialavori G, Biglieri A, et al. Estudio descriptivo de la situación nutricional en niños de 6-72 meses de la República Argentina. Resultados dela Encuesta Nacional deNutrición y Salud (ENNyS). Arch Argent Pediatr 2009;107(5):397-404.

10 Le Roy C, Reyes M, González JM, et al. Estado nutricional de vitamina $D$ en pre escolares chilenos de zonas australes. Rev Med Chil 2013;141(4):435-41.

11 Monangi N, Slaughter JL, Dawodu A, et al. Vitamin D status of early preterm infants and the effects of vitamin D intake during hospital stay. Arch Dis Child Fetal Neonatal Ed 2014;99(2):F166-8.

12 Fort P, Salas A, Nicola T, et al. A Comparison of 3 Vitamin D Dosing Regimens in Extremely Preterm Infants: A Randomized Controlled Trial. J Pediatr 2016;174:132-8.

13 Braegger C, Campoy C, Colomb V, et al. Vitamin D in the healthy European paediatric population. J Pediatr Gastroenterol Nutr 2013;56(6):692-701.

14 Cediel G, Corvalán C, Aguirre C, et al. Serum 25-Hydroxyvitamin D associated with indicators of body fat and insulin resistance in prepubertal Chilean children. Int J Obes (Lond) 2016;40(1):147-52.

15 CedielG,Corvalán C, López deRomañaD, etal.Prepubertal Adiposity, Vitamin D Status, and Insulin Resistance. Pediatrics 2016;138(1):e20160076.

16 Wortsman J, Matsuoka LY, Chen TC, et al. Decreased bioavailability of vitamin D in obesity. Am J Clin Nutr 2000;72(3):690-3.

17 Brinkmann K, Le Roy C, Iñiguez G, et al. Deficiencia severa de vitamina $D$ en niños de Punta Arenas, Chile: influencia de estado nutricional en la respuesta a suplementación. Rev Chil Pediatr 2015;86(3):182-8.

18 Płudowski P, Karczmarewicz E, Bayer M, et al. Practical guidelines for the supplementation of vitamin D and the treatment of deficitsin CentralEurope-recommended vitamin $\mathrm{D}$ intakes in the general population and groups at risk of vitamin D deficiency. Endokrynol Pol 2013;64(4):319-27.

19 Ding C, Wilding JPH, Bing C. 1,25-dihydroxyvitamin D3 protects against macrophage-induced activation of NFkB and MAPK signalling and chemokine release in human adipocytes. PLoS One 2013;8(4):e61707.

20 Belenchia AM, Tosh AK, Hillman LS, et al. Correcting vitamin D insufficiency improves insulin sensitivity in obese adolescents: a randomized controlled trial. Am JClin Nutr 2013;97(4):774-81.

21 Vitamin D supplement in early childhood and risk for Type I (insulin-dependent) diabetes mellitus. The EURODIAB Substudy 2 Study Group. Diabetologia 1999;42(1):51-4.

22 Hyppönen E, Läärä E, Reunanen A, et al. Intake of vitamin $\mathrm{D}$ and risk of type 1 diabetes: a birth-cohort study. Lancet 2001;358(9292):1500-3.

23 Mathieu C, Badenhoop K. Vitamin D and type 1 diabetes mellitus: state of the art. Trends Endocrinol Metab 2005;16(6):261-6.

24 Mohr SB, Garland CF, Gorham ED, et al. The association between ultraviolet $B$ irradiance, vitamin $D$ status and incidence rates of type 1 diabetes in 51 regions worldwide. Diabetologia 2008;51(8):1391-8.

25 Liu C, Lu M, Xia X, et al. Correlation of serum vitamin d level with type 1 diabetes mellitus in children: a metaanalysis. Nutr Hosp 2015;32(4):1591-4.

26 Ginanjar E, Sumariyono, Setiati S, et al. Vitamin D and autoimmune disease. Acta Med Indones 2007;39(3):133-41.

27 Reis JP, von Mühlen D, Miller ER, et al. Vitamin D status and cardiometabolic risk factors in the United States adolescent 
population. Pediatrics 2009;124(3):e371-9.

28 Hirschler V, Maccallini G, Sanchez M, etal. Improvement of Apolipoprotein B in Argentine Indigenous School Children after Vitamin DSupplementation. Cardiovasc Hematol Agents Med Chem 2015;13(2):137-45.

29 Hirschler V, Molinari C, Maccallini G, et al. Status of Dyslipidemia in Vitamin D Supplemented Argentinean Indigenous Children Versus A Non-supplemented Mixed Population Group. Cardiovasc Hematol Agents Med Chem 2015;13(2):129-36.

30 HirschlerV,MaccalliniG,TamboreneaMI,etal.Improvement in lipid profile after vitamin D supplementation in indigenous argentine school children. Cardiovasc Hematol Agents Med Chem 2014;12(1):42-9.

31 PyrżakB, Witkowska-SędekE, KrajewskaM, etal. Metabolic and immunological consequences of vitamin D deficiency in obese children. Adv Exp Med Biol 2015;840:13-9.

32 Dror Y, Giveon SM, Hoshen M, et al. Vitamin D levels for preventing acute coronary syndrome and mortality: evidence of a nonlinear association. J Clin Endocrinol Metab 2013;98(5):2160-7.

33 Al-Shoumer KA, Al-Essa TM. Is there a relationshipbetween vitamin $\mathrm{D}$ with insulin resistance and diabetes mellitus? World J Diabetes 2015;6(8):1057-64.

34 Margulies SL, Kurian D, Elliott MS, et al. Vitamin D deficiency in patients with intestinal malabsorption syndromes--think in and outside the gut. J Dig Dis 2015;16(11):617-33.

35 Norton L, PageS, Sheehan M, et al. Prevalence of inadequate vitamin d status and associated factors in children with cystic fibrosis. Nutr Clin Pract 2015;30(1):111-6.

36 Simoneau T, Bazzaz O, Sawicki GS, et al. Vitamin D Status in Children with Cystic Fibrosis. Associations with Inflammation and Bacterial Colonization. Ann Am Thorac Soc 2014;11(2):205-10.

37 Tangpricha V, Kelly A, Stephenson A, et al. An update on the screening, diagnosis, management, and treatment of vitamin $\mathrm{D}$ deficiency in individuals with cystic fibrosis: evidence-based recommendations from the Cystic Fibrosis Foundation. J Clin Endocrinol Metab 2012;97(4):1082-93.

38 Caruso R, Pallone F, Stasi E, et al. Appropriate nutrient supplementationin celiac disease. AnnMed 2013;45(8):522-31.

39 Tanpowpong P, Camargo CA. Early-life vitamin D deficiency and childhood-onset coeliac disease. Public Health Nutr 2014;17(4):823-6.

40 Capriles VD, Martini LA, Arêas JAG. Metabolic osteopathy in celiac disease: importance of a gluten-free diet. Nutr Rev 2009;67(10):599-606.

41 Le Roy OC, Rebollo GM, Moraga FM, et al. Nutrición del Niño con Enfermedades Neurológicas Prevalentes. Rev Chil Pediatr 2010;81(2):103-13.

42 Belderbos ME, Houben ML, Wilbrink B, et al. Cord blood vitamin D deficiency is associated with respiratory syncytial virus bronchiolitis. Pediatrics 2011;127(6):e1513-20.

43 Łuczyńska A, Logan C, Nieters A, et al. Cord blood 25(OH)
D levels and the subsequent risk of lower respiratory tract infections in early childhood: the Ulm birth cohort. Eur J Epidemiol 2014;29(8):585-94.

44 Esposito S, Lelii M. Vitamin D and respiratory tract infections in childhood. BMC Infect Dis 2015;15:487.

45 Urashima M, Segawa T, Okazaki M, etal. Randomized trial of vitamin D supplementation to prevent seasonal influenza A in schoolchildren. Am J Clin Nutr 2010;91(5):1255-60.

46 Tang JY, Epstein EH. Vitamin D and skin cancer. In: Feldman j, Pike W, Adams J. Vitamin D. 3rd ed. San Diego, CA:Elsevier;2011:1751-62. doi:10.1016/B978-0-12-3819789.10089-7.

47 Xiao L, Xing C, Yang Z, et al. Vitamin D supplementation for the prevention of childhood acute respiratory infections: a systematic review of randomised controlled trials. $\mathrm{Br} \mathrm{J}$ Nutr 2015;114(7):1026-34.

48 Einisman H, Reyes ML, Angulo J, al. Vitamin D levels and vitamin $D$ receptor gene polymorphisms in asthmatic children: a case-control study. Pediatr Allergy Immunol 2015;26(6):545-50.

49 Kerley CP, Hutchinson K, Cormican L, et al. Vitamin D3 for uncontrolled childhood asthma: A pilot study. Pediatr Allergy Immunol 2016;27(4):404-12. doi:10.

50 Villamor E, Marin C, Mora-Plazas M, et al. Vitamin D deficiency and age at menarche: a prospective study. Am J Clin Nutr 2011;94(4):1020-5.

51 Kao KT, Abidi N, Ranasinha S, et al. Low vitamin D is associated with hypertension in paediatric obesity. J Paediatr Child Health 2015;51(12):1207-13.

52 Davie M, Lawson DE. Assessment of plasma 25-hydroxyvitamin D response to ultraviolet irradiation over a controlled area in young and elderly subjects. Clin Sci (Lond) 1980;58(3):235-42.

53 Misra M, Pacaud D, Petryk A, et al. Vitamin D deficiency in children and its management: review of current knowledge and recommendations. Pediatrics 2008;122(2):398-417.

54 Holmlund-Suila E, Viljakainen $\mathrm{H}$, Hytinantti T, et al. Highdose vitamin $d$ intervention in infants--effects on vitamin d status, calcium homeostasis, and bone strength. J Clin Endocrinol Metab 2012;97(11):4139-47.

55 Oliveri B, Cassinelli H, Mautalen C, et al. Vitamin D prophylaxis in children with a single dose of $150000 \mathrm{IU}$ of vitamin D. Eur J Clin Nutr 1996;50(12):807-10.

56 Tachimoto H, Mezawa H, Segawa T, etal. Improved control of childhood asthma with low-dose, short-term vitamin D supplementation: a randomized, double-blind, placebocontrolled trial. Allergy 2016;71(7):1001-9.

57 Boucher BJ. The 2010 recommendations of the American Institute of Medicine for daily intakes of vitamin D. Public Health Nutr 2011;14(4):740.

58 Tau C, Ciriani V, Scaiola E, et al. Twice single doses of $100,000 \mathrm{IU}$ of vitamin D in winter is adequate and safe for prevention of vitamin D deficiency in healthy children from Ushuaia, Tierra Del Fuego, Argentina. J Steroid Biochem Mol Biol 2007;103(3-5):651-4. 